\title{
The Management of Irritable Bowel Syndrome: The Viewpoint of Italian General Practitioners in Activity and in Training
}

Bellini $\mathbf{M}^{1}$, Tosetti $C^{2}$, Rettura $F^{1 *}$, Morganti $\mathbf{R}^{3}$, Lambiase C1, Visaggi P1, Pancetti A', Benedetto E7, de Bortoli $N^{1}$, Marchi $S^{1}$ and De Bastiani $\mathbf{R}^{2}$ ${ }^{1}$ Department of Translational Research in Medicine and Surgery, University of Pisa, Italy

${ }^{2}$ Italian Group for Primary Care Gastroenterology, General Practitioner and Gastroenterologist, National Health Service, Italy

${ }^{3}$ Clinical Trial Statistical Support Unit, Azienda Ospedaliero Universitaria Pisana, Italy

*Correspondling author: Rettura F, Department of Translational Research in Medicine and Surgery, University of Pisa-Ospedale Cisanello, Gastroenterology Unit, Via P. Trivella, 56124 Pisa, Italy

Received: J une 16, 2021; Accepted: July 02, 2021; Published: July 09, 2021

\begin{abstract}
Background: Guidelines on Irritable Bowel Syndrome (IBS) are usually developed by specialists, with a possible translational gap in primary care.

Aims: To evaluate the knowledge of IBS of Italian General Practitioners (GPs) and the difference compared to doctors in Training for General Practice (ITGPS).

Methods: A questionnaire was completed by 170 GPs and 64 ITGPs.

Results: The Rome Criteria and Bristol Scale are more familiar to ITGPS than GPs. The most frequently used diagnostic symptoms are abdominal pain, bowel movement frequency and bloating. GPs and ITGPs think that bloating and abdominal discomfort should be introduced into the definition of IBS. Intestinal motility disorders and psychological factors are considered to be the most probable cause of IBS. GPS report more frequently than ITGPS that the patient's request and difficulties in managing the disorder are reasons for a gastroenterological referral.
\end{abstract}

Conclusions: There is still a gap between the indications provided by the experts and clinical practice, and this seems greater on the part of GPs than ITGPs. Abdominal pain and bowel frequency changes are considered the main symptoms for diagnosing IBS, but most GPS and ITGPs would like to include bloating among the diagnostic criteria. It would be highly desirable to develop commonly shared guidelines between gastroenterologists and GPs.

Keywords: Irritable bowel syndrome; General practitioners; Primary care

\section{Introduction}

Irritable Bowel Syndrome(IBS) represents one of the most frequent gastroenterological clinical problems both for specialists and General Practitioners (GPs) [1-5]. As for the other functional disorders, the complex and multifaceted pathophysiological aspects often lead to iteration of unnecessary medical examinations and diagnostic tests and to a multiplicity of therapeutic suggestions, which are sometimes contradictory and conflicting. It is therefore not surprising that the management of IBS patients is frequently unsatisfactory [6,7]. The IBS definition itself is still under discussion, so even the diagnostic criteria internationally proposed by gastroenterologists, such as the Rome IV Criteria (RC) [8], are often scarcely used in clinical practice by gastroenterologists themselves, even if they remain a cornerstone of research studies [9]. In addition, since most of the guidelines and position papers published on this topic have been conceived within a gastroenterological setting, it is likely that a translational "gap" exists with primary care, possibly inducing a certain inertia both in the diffusion and in the acceptance of such criteria by the GPs [10]. This is even more important in the Italian primary care setting where most GPs have had many years of activity. This means that they could very well be anchored to their professional routine, or simply resistant to changes, also considering that in the last ten years there have been no decisive and substantial changes in the diagnostic and therapeutic approach to IBS. Conversely, young doctors still in Training for General Practice (ITGPs), who are partially included in an educational circuit in close connection also with specialists, could be provided with more up-to-date knowledge and could have these concepts integrated into their professional practice.

The aim of the present study was to evaluate the knowledge and the use of the diagnostic and management criteria for IBS in a group of Italian GPs and ITGPs.

\section{Materials and Methods}

The survey was carried out in October 2019 in three cities located in the North, in the Center and in the South of Italy. An invitation to participate in the study was sent to 300 GPs randomly selected from the lists and 90 ITGPs attending the final year of their course.

The invitation was accompanied by the description of the purpose of the survey and the link to an online form. The form was totally anonymous without any possibility of identifying the participants. The access to the form was authorized only upon an express declaration of consent to participate.

The form contained demographic information such as age, gender, years since graduation ( $\leq 20$ years $v s .>20$ years) and number of patients $(\leq 1000$ vs. $>1000)$ under their care. Respondents were
J Fam Med - Volume 8 Issue 6 - 2021

ISSN : 2380-0658 | www.austinpublishing group.com

Rettura et al. (C) All rights are reserved
Citation: Bellini M, Tosetti C, Rettura F, Morganti R, Lambiase C, Visaggi P, et al. The Management of Irritable Bowel Syndrome: The Viewpoint of Italian General Practitioners in Activity and in Training. J Fam Med. 2021; 8(6): 1264. 
required to answer about their knowledge and use of the $\mathrm{RC}$ and of the Bristol Scale (BS) for the determination of fecal consistency, and on the symptoms considered fundamental for the diagnosis of IBS. They also had to assess (improbable $v s$. probable) the relevance of the possible causes of IBS and the reasons for the possible referral to a gastroenterologist.

Respondents were finally required to rate the percentage of patients in whom satisfactory IBS management defined as 'a patient who perceives her/his symptoms as no more than a nuisance and rejoins that silent majority of people with IBS who do not seek health care' [10] could be achieved, make a judgement about their overall knowledge of IBS (satisfactory/unsatisfactory) and assess their needs for further specific professional education (yes/no).

The investigation was carried out according to the principles of the Helsinki Declaration. The approval of an Ethics Committee was not required, according to national legislation, given that this was an anonymous survey among professionals.

\section{Statistical methods}

Categorical data were described by absolute and relative frequency, continuous data by mean and standard deviation. To analyze qualitative and quantitative factors the chi square test or z-test for two proportions and t-test for independent samples (two tailed) were performed, respectively. Multivariate models based on logistic regression were applied to compare independent variables with one specific dependent variable. Significance was fixed at 0.05 . All analyzes were carried-out by SPSS vs. 26 technology.

\section{Results}

One hundred and seventy GPs (56.7\%) and 64 ITGPs (71.1\%) participated in the survey. The two groups showed significant differences not only with respect to age $(\mathrm{p}=0.001)$, years since graduation $(\mathrm{p}=0.001)$ and specialization in gastroenterological disciplines $(p=0.002)$, but also as regards their distribution by sex, the ITGPs being more frequently female than the respondent GPs $(\mathrm{p}=0.001)$ (Table 1).

The ITGPs were more frequently familiar than the GPs with both the RC $(73.4 \%$ vs. $47.6 \% ; \mathrm{p}=0001)$ and $\mathrm{BS}(70.3 \%$ vs. $46.5 \% ; \mathrm{p}=0.002)$.

Gender and years since graduation did not affect the GPs' knowledge of RC and BS, but GPs with a gastroenterology specialization were more frequently familiar with the RC and BS compared to GPs without this specialization (respectively $76.9 \%$ vs. 42.4\%; $\mathrm{p}=0.001$; and $65.4 \%$ vs. $43.1 \%$; $\mathrm{p}=0.036$ ).

Among the GPs familiar with the RC, $86.4 \%$ considered them to be acceptable and $76.5 \%$ declared that they used them in clinical practice, whereas among the ITGPs familiar with the RC, $89.4 \%$ reported them to be acceptable and $74.8 \%$ used them in clinical practice; so no significant differences were observed between GPs and ITGPs.-

Among the participants having knowledge of the BS, GPs considered it more useful in clinical practice than ITGPs $(81.0 \%$ vs. 62.2\%; $\mathrm{p}=0.037$ ).

Among the participants familiar with the RC, $63.0 \%$ of the GPs and $44.7 \%$ of the ITGPs ( $\mathrm{p}=0.017$ ) would introduce other symptoms into the IBS definition provided by the RC: abdominal bloating $(80.4 \%$ of the GPs vs. $95.2 \%$ of ITGPs; $\mathrm{p}=0.01$ ) and abdominal discomfort ( $43.1 \%$ of the GPs $v s .45 \%$ of the ITGPs; ns) (Table 2).

Abdominal pain related to defecation and changes in the frequency of bowel movements were the most used symptoms for the diagnosis of IBS in clinical practice, both by the GPs and the ITGPs, but with some differences between them. Indeed, more ITGPs than GPs said that they used "abdominal pain" ( $90.6 \%$ vs. $76.5 \%$; $=0.025)$

Table 1: Main features of the GPs and the ITGPs answering the survey.

\begin{tabular}{|l|c|c|}
\hline & GPs (n= 170) & ITGPs (n=64) \\
\hline Male/female & $118 / 52$ & $20 / 44$ \\
\hline Age, mean (SD), years & $60.0(7.6)$ & $31.2(4.2)$ \\
\hline$>$ 20 years of graduation (\%) & $155(91,2)$ & 0.001 \\
\hline Number of registered (under care) patients, mean (SD) & $1294.2(345.2)$ & 0.001 \\
\hline Specialization in gastroenterology (\%) & $26(15.3)$ & 0.001 \\
\hline
\end{tabular}

GPs: General Practitioners; ITGPs: in-Training General Practitioners.

Table 2: Awareness and use of the RC and the BS.

\begin{tabular}{|c|c|c|c|}
\hline & GPs $(n=170)$ & ITGPs (n=64) & $P$ Value \\
\hline Awareness of the RC (\%) & $81(47.6)$ & $47(73.4)$ & 0.001 \\
\hline Use of the RC in the current practice (\%)* & $62 / 81(76.5)$ & $35 / 47(74.8)$ & n.s. \\
\hline Approval of the definition of IBS of the RC $(\%)^{*}$ & $70 / 81(86.4)$ & $42 / 47(89.4)$ & n.s. \\
\hline Willingness to introduce other symptoms into the definition of IBS of the RC (\%) & $51 / 81(63.0)$ & $21 / 47(44.7)$ & n.s. \\
\hline Introduction of the symptom "abdominal bloating" into the definition of IBS $(\%)^{\#}$ & $41 / 51(80.4)$ & 20/21 (95.2) & n.s. \\
\hline Introduction of the symptom "abdominal discomfort" or annoyance" into the definition of IBS $(\%)^{\#}$ & 22/51 (43.1) & $9 / 21(45.0)$ & n.s. \\
\hline Awareness of the BS (\%) & $79(46.5)$ & $45(70.3)$ & 0.002 \\
\hline Use of the BS in current practice $(\%)^{\mathrm{c}}$ & $64 / 79(81.0)$ & $28 / 45(62.2)$ & 0.037 \\
\hline
\end{tabular}

GPS: General Practitioners; ITGPs: in-Training General Practitioners; RC: Rome Criteria; BS: Bristol Scale; IBS: Irritable Bowel Syndrome.

*Among physicians who had stated that they are familiar with the RC.

"Among physicians who had stated that they want to introduce other symptoms into the definition of IBS provided by the RC.

Among physicians who had stated that they are familiar with the BS. 
Table 3: Most frequent symptoms used in clinical practice for the diagnosis of IBS

\begin{tabular}{|c|c|c|c|}
\hline & GPs $(n=170)$ & ITGPs $(n=64)$ & P Value \\
\hline Changes in the frequency of bowel movements (\%) & $107(62.9)$ & $56(87.5)$ & 0.001 \\
\hline Abdominal pain related to defecation (\%) & $130(76.5)$ & $58(90.6)$ & 0.025 \\
\hline Difficult or incomplete defecation (\%) & $49(28.8)$ & $25(39.1)$ & n.s. \\
\hline Rectal mucus emission (\%) & $27(15.9)$ & $9(14.0)$ & n.s. \\
\hline Abdominal bloating (\%) & $112(65.9)$ & $46(71.9)$ & n.s. \\
\hline Defecatory urgency (\%) & $24(14.1)$ & $22(34.4)$ & 0.001 \\
\hline
\end{tabular}

GPS: General Practitioners; ITGPS: in-Training General Practitioners; IBS: Irritable Bowel Syndrome.

Table 4: Pathophysiological mechanisms of IBS: frequency of "probable" answers.

\begin{tabular}{|l|c|c|c|}
\hline & GPs (n= 170) & ITGPs (n=64) & P Value \\
\hline Intestinal motility disorders (\%) & $110(64.7)$ & $41(64.1)$ & n.s. \\
\hline Food allergy and/or intolerance (\%) & $79(46,5)$ & $29(45.3)$ & n.s. \\
\hline Psychological factors (\%) & $104(61.2)$ & $40(62.5)$ & n.s. \\
\hline Gut infections (\%) & $82(48.2)$ & $34(54.7)$ & n.s. \\
\hline Visceral hypersensitivity (\%) & $85(50.0)$ & $35(54.7)$ & n.s. \\
\hline Gut microbial dysbiosis (\%) & $97(57.1)$ & $38(59.4)$ & n.s. \\
\hline
\end{tabular}

GPS: general practitioners; ITGPS: in-training general practitioners; IBS: Irritable Bowel Syndrome.

and "changes in the frequency of bowel movements" (87.5 \%\% vs. $62.9 \% ; \mathrm{p}=0.001$ ). Also bloating was frequently reported but without significant differences between the GPs (65.9\%) and the ITGPs (71.9\%). Difficult or incomplete defecation, rectal mucus emission and defecatory urgency were cited by less than $40 \%$ of both GPs and ITGPs, the latter, however, being indicated more by ITGPs than by GPs (34.4\% vs. 14.1\%; $\mathrm{p}=0.001)$. Gender and years since graduation did not affect the identification of diagnostic symptoms by the GPs, but the use of "abdominal pain related to defecation" in order to reach an IBS diagnosis was greater among the GPs with a gastroenterological specialization compared to the GPs without this specialization $(92.3 \%$ vs. 73.6. \%, $\mathrm{p}=0.039$ ) (Table 3).

The prevalence of "probable" answers about the different pathogenetic hypotheses are reported in Table 4. The causes considered most probable $(>60 \%)$ were the intestinal motility disorders and the psychological factors, without any difference between GPs and ITGPs. Gut dysbiosis, visceral hypersensitivity, gut infections and food allergy/intolerance were considered a "probable" hypothesis by less than $60 \%$ of the participants without any difference between the GPs and the ITGPs.

Some differences deserve to be highlighted regarding demographic factors. The GPs with less than 20 years since graduation thought that the hypothesis of psychological disorders was more probable than the GPs with more than 20 years since graduation $(81.7 \%$ vs. $58.7 \%$; $\mathrm{p}=0.047)$. The GPs with a gastroenterological specialization considered the hypothesis of the abnormalities of gut motility to be more frequently probable than GPs without the specialization ( $88.5 \%$ vs. 60.4\%; $\mathrm{p}=0.011$ ). Moreover, the female ITGPs declared the hypothesis of psychological disorders to be more frequently probable than male ITGPs ( $80.0 \%$ vs. $50.0 \%$; $\mathrm{p}=0.012)$.

The frequency of the different reasons for a gastroenterological referral are reported in Table 5. Therapeutic failure, the performance
Table 5: Reasons for a gastroenterological referral: frequency of "probable" answers.

\begin{tabular}{|l|c|c|c|}
\hline & GPs (n= 170) & ITGPs (n=64) & P Value \\
\hline Patient's request (\%) & $92(54.1)$ & $22(34.4)$ & 0.011 \\
\hline Therapeutic failure (\%) & $100(60.0)$ & $45(70.3)$ & n.s. \\
\hline Second level diagnostic tests (\%) & $97(57.1)$ & $41(64.1)$ & n.s. \\
\hline Patient's reassurance (\%) & $98(57.6)$ & $34(53.1)$ & n.s. \\
\hline Difficulties in management (\%) & $97(57.1)$ & $9(14.1)$ & 0.001 \\
\hline
\end{tabular}

GPs: General Practitioners; ITGPs: in-Training General Practitioners.

Table 6: Educational needs regarding IBS

\begin{tabular}{|l|c|c|c|}
\hline & $\begin{array}{c}\text { GPs } \\
(\mathbf{n = 1 7 0 )}\end{array}$ & $\begin{array}{c}\text { ITGPs } \\
(\mathbf{n = 6 4 )}\end{array}$ & P Value \\
\hline $\begin{array}{l}\text { Knowledge of IBS is considered } \\
\text { satisfactory (\%) }\end{array}$ & $79(46.5)$ & $45(70.3)$ & 0.002 \\
\hline $\begin{array}{l}\text { An update on IBS is considered useful } \\
(\%)\end{array}$ & $64(37.6)$ & $28(43.8)$ & n.s. \\
\hline
\end{tabular}

GPS: General Practitioners; ITGPs: in-Training General Practitioners; IBS Irritable Bowel Syndrome.

of "second level" diagnostic tests and the need to reassure the patient were very frequently reported both by GPs and ITGPs without any significant differences between the two groups.

Conversely, two answers were more frequently given by the GPs compared to the ITGPs: patient's request $(54.1 \%$ vs. $34.4 \% ; \mathrm{p}=0.011)$ and difficulties in management $(57.1 \%$ vs. $14.1 \%$; $=0.001)$. With respect to demographic factors, GPs with more than 1000 patients considered the hypothesis of "reassuring the patient" to be more likely as a cause of referral compared to GPs with less than 1000 patients (65.2\% vs. $42.9 \%$; $\mathrm{p}=0.018)$.

The aim of achieving a satisfactory patient management ("coexistence with symptoms") was obtained by $52.9 \%$ of ITGPs compared to $41.3 \%$ of GPs $(\mathrm{p}=0.005)$, without any demographic difference within the two groups.

Regarding educational needs, the ITGPs reported that their knowledge of IBS was satisfactory more frequently than that indicated by the GPs (70.3\% vs. $46.5 \%$; $\mathrm{p}=0.002)$. GPs with a gastroenterology specialization considered their knowledge of IBS to be satisfactory more frequently than GPs without this specialization $(65.4 \%$ vs. $29.9 \% ; \mathrm{p}=0.001)$. The number of those considering a professional update on IBS useful was not significantly different between GPs (37.6\%) and ITGPs (43.8\%) (Table 6).

\section{Discussion}

The results of this survey provide important information on the attitude of both Italian GPs and ITGPs in managing IBS. The two 
groups are, obviously, markedly different in terms of age, seniority, and gastroenterology specialization. Moreover, most of the GPs are males, whereas among ITGPs there is a greater prevalence of females. This reflects the Italian situation where there is a higher number of female ITGPs than male ITGPs.

The knowledge of the RC for IBS among GPs is low, although it is increasing if compared to the results of a survey we carried out in 2005 (47.6\% vs. 35.7\%). Also their use in clinical practice by those who know the RC has increased $(76.5 \%$ vs. 60\%) [10]. However, these results are comparable to those obtained by similar studies carried out in different countries. These confirm that the knowledge and the routine use of these criteria are widespread only among gastroenterologists with a particular aptitude for managing functional digestive disorders [11-15]. On the other hand, the criteria are scarcely known and used in primary care where most patients are diagnosed and treated $[9,11,14-16]$.

IBS positive diagnostic criteria are largely based on expert opinion and not on high quality evidence. Very few data report their applicability in primary care [17] and very few studies attempted to validate a positive diagnostic strategy for IBS in primary care. Begtrup et al. showed that a positive strategy was not inferior to an exclusion strategy, with lower direct costs and less use of health resources, and yet with similar effects on symptoms and patients' satisfaction [18]. However, at present, worldwide, the diagnosis of IBS still largely remains an exclusion diagnosis for GPs $[15,19,20]$. It is a complex procedure requiring a non negligible amount of time and high costs [19].

On the contrary, there is a higher percentage of ITGPs, more recently graduated, who are more familiar with the RC than the GPs, demonstrating that the awareness of the RC has been constantly increasing in recent years. Hence, even if among those familiar with the RC the percentage of GPS and ITGPs using them in clinical practice is not significantly different, the RC end up being used more by ITGPs than GPs (respectively $54.7 \%$ vs. $36.5 \%$; $\mathrm{p}=0.017$ ).

In addition, the knowledge of the BS is more widespread among ITGPs, but the GPs use it more frequently than the ITGPs in their clinical practice, probably because they find the BS rapid and easily understandable by patients due to its visual immediacy.

As expected, more GPs with a gastroenterological specialization are familiar with both the RC and BS than other colleagues, but this does not imply a significant difference in their use in clinical practice.

A large number of the respondents feel that other symptoms should be added to the definition of IBS ( $63 \%$ of GPs and $44.7 \%$ of ITGPS) in order to achieve a correct clinical picture of IBS patients. Both GPs and ITGPs would like to include abdominal bloating (80.4\% of GPs and $95.2 \%$ of ITGPs) among the diagnostic criteria for IBS. With a lower percentage they think that also abdominal discomfort (43.1\% of GPs and $45 \%$ of ITGPs), present in RC III but eliminated from the new RC IV, is an important symptom. GPs with a gastroenterological specialization would like to include bloating among diagnostic IBS criteria more frequently $(\mathrm{p}=0.018)$ than GPs without gastroenterological specialization. The degree of seniority and the number of patients under their care do not seem to make any difference among the GPs, together with gender, which does not influence any choice of either GPs or ITGPs.

As reported in our previous survey, abdominal pain and changes in the frequency of bowel movements are symptoms frequently used in clinical practice by GPs [10]. Based on our results, it seems that ITGPs use them even more frequently than GPs. Abdominal bloating, which had been considered an important symptom for the diagnosis of IBS also by $78.6 \%$ of the GPs who participated in the 2005 survey [10], is still frequently used both GPs and ITGPs with no significant difference between them.

Bloating is considered as only an additional diagnostic criterion by RC, although it is often reported as the most bothersome symptom by patients and GPs [21], and is the third most frequent IBS symptom according to GPs and gastroenterologists [11].

In the NICE guidelines, bloating was only a supportive element [22] but in the 2015 revision it became one of the basic symptoms [23] and the Consensus development using nominal group technique created for European primary care gastroenterology identifies the defining features of IBS in: alteration of bowel habits, bloating and abdominal pain (or discomfort or annoyance) [24]. Abdominal bloating in IBS is associated with increased symptoms and pain severity, somatization, depression, fibromyalgia, and altered dietary fluid composition. Recognizing and addressing these factors in the diagnosis and management of patients with IBS may improve clinical outcome [25]. It is interesting to underline that regarding abdominal bloating even younger doctors (ITGPs) have the same opinion as their older colleagues (GPs), whereas ITGPs seem to pay more attention to defecatory problems because they use defecatory urgency as a diagnostic symptom more frequently than GPs.

Among GPs, those with a gastroenterological specialization recognize abdominal pain even more frequently as a pivotal symptom. As reported above, gender does not influence any choices among both GPs and ITGPs. Among GPs, neither seniority nor number of patients under their care seems to influence any choice.

As regards the pathogenetic hypotheses, alterations of intestinal motility and psychological factors remain the most frequently considered causes, as detected in our previous investigation [10]. This is without any difference between GPs and ITGPs, but also other factors (e.g. gut dysbiosis and infections, visceral hypersensitivity, and food allergy/intolerance) are considered among the most frequent pathophysiological mechanisms. These data confirm the results data coming from previous studies showing broad areas of uncertainty among GPs about the etiology of IBS [26]. Most GPs give importance to psychological factors and stress $[9,15,19,26,27]$, but there is a nonunequivocal opinion regarding the role of other possible factors $[15,21,27]$. As a matter of fact, IBS pathogenesis is one of the most debated and controversial issues in the gastroenterological field and many different, sometimes conflicting, hypotheses have been put forward [6].

With regard to the reasons for referring the patients to a gastroenterologist, the GPs report different motivations (i.e. the difficulty of management, the need for an in-depth diagnostic pathway and the need to reassure the patient). The proportion of GPs requiring specialist consultation varies widely between countries, with differing motivations (probably due to the different organization of 
the local National Health System) [15,28-30]. As reported by Mujagic et al., a specialist referral can be useful to reassure patients whose symptoms partially respond to the GP's therapy and/or to improve patients' satisfaction [9]. In one study published in 1997, male and older doctors referred patients to a gastroenterologist more than female and younger doctors [31], whereas in our previous survey no difference was observed regarding specialist referral between older and younger doctors and between male and female doctors [10].

In the present survey GPs reported more frequently than ITGPs that factors inducing a specialist consultation were the patient's request and the difficulties in management. This data must be read together with the evaluation of satisfactory patient management obtainable according to the ITGPs in more than $50 \%$ of patients and for GPs only in about $40 \%$. It is a quite different result from that obtained (62.7\%) in our previous investigation [10]. Particularly, the referral to a gastroenterologist due to a patient's request is reported in highly variable percentages $[10,32]$. A study recently carried out in Spain has shown that IBS patients would prefer to be referred to a gastroenterologist more frequently than their GPs usually do so, even if the same study highlighted that there was no difference in the frequency of patients satisfied with the care provided by their GP or by the gastroenterologist [33]. The patients perceived reluctance by GPs in asking for a gastroenterological consultation [33]. Indeed, referral is a sensitive topic for GPs, involving emotionally charged interactions and relationships with patients, colleagues, specialists and supervisors. Consequentially, the decision to refer or not is influenced by multiple environmental, personal and clinical factors which dynamically interact and affect the decision-making process $[9,34]$.

Finally, as far as training needs are concerned, the ITGPs, recently graduated, believe that their personal knowledge of IBS is satisfactory more frequently than the GPs, even if the percentage of respondents who deem a specific professional update to be useful is around $40 \%$, without differences between GPs and ITGPs. Compared to our previous investigation [10], the percentage of GPs considering their knowledge sufficient is remarkably similar, but the percentage of the GPs regarding a professional update as useful has more than tripled. Among GPs no correlation between seniority of degree and number of patients was observed regarding these variables, but GPs with a gastroenterology specialization consider more often that they have a satisfactory knowledge of IBS.

The results of this survey clearly show that it is mandatory to constantly update the knowledge of GPs and ITGPs about IBS. There is still a gap between the indications provided by the experts, i.e. national and international guidelines, and local practice. This gap has persisted over the years, probably indicating an insufficient communication between the various professional healthcare figures. Gastroenterologists do not fully convince GPs and perhaps GPs, conversely, fail to adequately express the reality they face every day, together with their needs and requirements. It could also be highly desirable to develop common guidelines in order to share management paths between gastroenterologists and GPs. This could reduce the number of unnecessary diagnostic tests and gastroenterological consultations, resulting in a higher rate of patients' satisfaction, and enabling an alternative and more effective allocation of health expenditure. This aim could be more easily achieved if also ITGPs, the younger doctors still in training to become GPs, were actively involved.

\section{Acknowledgement}

The authors are grateful to the following GPs of the Italian Group for Primary Care Gastroenterology (GIGA-CP) for their valuable help in carrying out the study: Alessandra Belvedere, Alberto Bozzani, Giovanni Casella, Maurizio Mancuso, Luigi Napoli, Enzo Pirrotta, Riccardo Scoglio, Enzo Ubaldi and to Chris Powell for the language revision of this paper.

\section{Conflicts of Interest Statement}

The authors certify that they have NO affiliations with or involvement in any organization or entity with any financial interest (such as honoraria; educational grants; participation in speakers' bureaus; membership, employment, consultancies, stock ownership, or other equity interest; and expert testimony or patent-licensing arrangements), or non-financial interest (such as personal or professional relationships, affiliations, knowledge or beliefs) in the subject matter or materials discussed in this manuscript.

\section{References}

1. Gladman LM, Gorard DA. General practitioner and hospital specialist attitudes to functional gastrointestinal disorders. Aliment Pharmacol Ther. 2003; 17: 651-654.

2. Palsson OS, Whitehead W, Törnblom $\mathrm{H}$, et al. Prevalence of Rome IV Functional Bowel Disorders Among Adults in the United States, Canada, and the United Kingdom. Gastroenterology. 2020; 158: 1262-1273.e3.

3. Sperber AD, Bangdiwala SI, Drossman DA, et al. Worldwide Prevalence and Burden of Functional Gastrointestinal Disorders, Results of Rome Foundation Global Study. Gastroenterology. 2021; 160: 99-114.e3.

4. Creed F. Review article: the incidence and risk factors for irritable bowel syndrome in population-based studies. Aliment Pharmacol Ther. 2019; 50: 507-516.

5. Thompson WG, Heaton KW, Smyth GT, et al. Irritable bowel syndrome in general practice: prevalence, characteristics, and referral. Gut. 2000; 46: 7882.

6. Bellini M, Gambaccini D, Stasi C, et al. Irritable bowel syndrome: a disease stil searching for pathogenesis, diagnosis and therapy. World J Gastroenterol. 2014; 20: 8807-8820.

7. Tack J, Stanghellini V, Mearin F, et al; IBIS-C Study group. Economic burden of moderate to severe irritable bowel syndrome with constipation in six European countries. BMC Gastroenterol. 2019; 19: 69.

8. Mearin F, Lacy BE, Chang L, et al. Bowel Disorders. Gastroenterology. 2016 S0016-5085: 00222-00225.

9. Mujagic $Z$, Jonkers DMAE, Hungin APS, et al. Use of Rome criteria for the diagnosis of irritable bowel syndrome in primary care: a survey among European countries. Eur J Gastroenterol Hepatol. 2017; 29: 651-656.

10. Bellini M, Tosetti C, Costa F, et al. The general practitioner's approach to irritable bowel syndrome: from intention to practice. Dig Liver Dis. 2005; 37: 934-939.

11. Olafsdottir LB, Gudjonsson H, Jonsdottir HH, et al. Irritable bowel syndrome: physicians' awareness and patients' experience. World J Gastroenterol. 2012; 18: 3715-3720.

12. Charapata C, Mertz H. Physician knowledge of Rome symptom criteria for irritable bowel syndrome is poor among non-gastroenterologists. Neurogastroenterol Motil. 2006; 18: 211-216.

13. Andresen $V$, Whorwell $P$, Fortea $J$, et al. An exploration of the barriers to the confident diagnosis of irritable bowel syndrome: A survey among genera practitioners, gastroenterologists and experts in five European countries. 
United European Gastroenterol J. 2015; 3: 39-52.

14. Gladman LM, Gorard DA. General practitioner and hospital specialis attitudes to functional gastrointestinal disorders. Aliment Pharmacol Ther 2003; 17: 651-654

15. Hungin AP, Molloy-Bland M, Claes R, et al. Systematic review: the perceptions, diagnosis and management of irritable bowel syndrome in primary care--a Rome Foundation working team report. Aliment Pharmaco Ther. 2014; 40: 1133-1145.

16. Al-Shamrani HAA, Khalil H, Khan MS. Awareness and Utilization of ROME Criteria for Diagnosis of Inflammatory Bowel Syndrome among Primary Care Physicians in Riyadh, Saudi Arabia. Mater Sociomed. 2020; 32: 112-116.

17. Talley NJ. Commentary: Controversies in NICE guidance on irritable bowe syndrome. BMJ. 2008; 336: 558-559.

18. Begtrup LM, Engsbro AL, Kjeldsen J, et al. A positive diagnostic strategy is noninferior to a strategy of exclusion for patients with irritable bowel syndrome. Clin Gastroenterol Hepatol. 2013; 11: 956-962.e1.

19. Harkness EF, Harrington V, Hinder S, et al. GP perspectives of irritable bowel syndrome--an accepted illness, but management deviates from guidelines: a qualitative study. BMC Fam Pract. 2013; 14: 92.

20. Spiegel BM, Farid M, Esrailian E, Talley J, Chang L. Is irritable bowel syndrome a diagnosis of exclusion?: a survey of primary care providers, gastroenterologists, and IBS experts. Am J Gastroenterol. 2010; 105: 848858.

21. Bijkerk CJ, de Wit NJ, Stalman WA, et al. Irritable bowel syndrome in primary care: the patients' and doctors' views on symptoms, etiology and management. Can J Gastroenterol. 2003; 17: 363-368; 405-406.

22. Dalrymple J, Bullock I. Diagnosis and management of irritable bowel syndrome in adults in primary care: summary of NICE guidance. BMJ. 2008 336: 556-558.

23. NICE clinical guideline 61. Irritable bowel syndrome in adults: diagnosis and management of irritable bowel syndrome in primary care. 2015.

24. Rubin G, De Wit N, Meineche-Schmidt V, et al. The diagnosis of IBS in primary care: consensus development using nominal group technique. Fam Pract. 2006; 23: 687-692.
25. Hod K, Ringel Y, van Tilburg MAL, et al. Bloating in Irritable Bowel Syndrome Is Associated with Symptoms Severity, Psychological Factors, and Comorbidities. Dig Dis Sci. 2019; 64: 1288-1295.

26. Bradley S, Alderson S, Ford AC, et al. General practitioners' perceptions of irritable bowel syndrome: a Q-methodological study. Fam Pract. 2018; 35: 74-79.

27. Seifert B, Rubin G, de Wit N, et al. The management of common gastrointestinal disorders in general practice A survey by the European Society for Primary Care Gastroenterology (ESPCG) in six European countries. Dig Liver Dis. 2008; 40: 659-666.

28. Lacy BE, Rosemore J, Robertson D, et al. Physicians' attitudes and practices in the evaluation and treatment of irritable bowel syndrome. Scand J Gastroenterol. 2006; 41: 892-902.

29. Smith GD, Steinke DT, Kinnear M, et al; Episode IBS study. A comparison of irritable bowel syndrome patients managed in primary and secondary care: the Episode IBS study. Br J Gen Pract. 2004; 54: 503-507.

30. Bellini M, Tosetti C, Stasi C, et al. The general practitioner's management of patients with a new diagnosis of irritable bowel syndrome. J Clin Gastroenterol. 2006; 40: 87

31. Thompson WG, Heaton KW, Smyth GT, et al. Irritable bowel syndrome: the view from general practice. Eur J Gastroenterol Hepatol. 1997; 9: 689-692.

32. Franke A, Singer MV, Dumitraşcu DL. How general practitioners manage patients with irritable bowel syndrome. Data from a German urban area. Rom J Intern Med. 2009; 47: 47-53.

33. Mira JJ, Guilabert M, Sempere L, et al. The irritable bowel syndrome care process from the patients' and professionals' views. Rev Esp Enferm Dig. 2015; 107: 202-210.

34. Tzartzas K, Oberhauser PN, Marion-Veyron R, et al. General practitioners referring patients to specialists in tertiary healthcare: a qualitative study. BMC Fam Pract. 2019; 20: 165 